\title{
Vehicle Trajectory Impacts of Adaptive Cruise Control
}

\author{
Andreas Tapani*
}

\begin{abstract}
Adaptive Cruise Control (ACC) is assumed to have a potential to improve quality-of-service and safety and to reduce the environmental impact of the road traffic system. This paper use vehicle trajectories from traffic simulation to study impacts of ACC on vehicle acceleration and deceleration rates. The analysis is based on traffic simulations with car-following models including ACC functionality and driver behaviour in ACC-equipped as well as standard non-equipped vehicles. The simulation results show that ACC can improve the traffic situation in terms of reduced acceleration and deceleration rates even though macroscopic traffic properties may remain uninfluenced. This supports the hypothesised positive road safety and environmental effects of ACC. It is also established that the results are largely dependent on the assumptions made regarding driver behaviour in ACC-equipped and standard vehicles. It is consequently crucial to include appropriate assumptions regarding driver behaviour in traffic simulation based analyses of ACC.
\end{abstract}

\section{Introduction}

The last decade has seen an increase in the interest in Intelligent Transportation Systems (ITS) to improve quality-of-service and safety and to reduce the negative environmental impact of road traffic. One category of ITS that is expected to have substantial impact on future road traffic is in-vehicle Advanced Driver Assistance Systems (ADAS) (Berghout

${ }^{*}$ Swedish National Road and Transport Research Institute (VTI) and Linköping University, Department of Science and Technology (ITN), AdDress: VTI, SE-581 95 Linköping, Sweden, TEL: +46 132042 80, E-MAIL: andreas.tapani@vti.se 
et al., 2003). The first generation of ADAS that is currently commercially available includes stand alone systems such as adaptive cruise controls, speed limiters and lane-keeping assistants. Co-operative systems based on vehicle-to-vehicle communication are expected to be introduced in the future.

Possible benefits provided by ADAS are determined by the properties of the ADAS-equipped driver/vehicle unit and its interactions with the surrounding traffic. Traffic micro-simulation models that consider individual vehicles in the traffic stream are consequently useful tools for traffic system impact analysis of ADAS. These models allow inclusion of ADAS functionalities and the induced driver behaviour in driver/vehicle sub-models of the simulation. Traffic impacts of the considered ADAS can then be estimated through simulations including ADAS-equipped vehicles (see e.g. Hoogendoorn and Minderhoud, 2002; Liu and Tate, 2004; Tapani, 2008).

Adaptive Cruise Control (ACC) is among the most studied ADAS. Several driver behavioural studies of driving with ACC have been performed (Dragutinovic et al., 2005; Saad et al., 2004). ACC has also been the subject of many traffic simulation experiments (Minderhoud and Bovy, 1999; Hoogendoorn and Minderhoud, 2002; Davis, 2004, 2007; van Arem et al., 2006; Kesting et al., 2007a, 2007b). Most traffic simulation studies of ACC impacts have focused on macroscopic capacity and quality-of-service impacts of the distance keeping functionality of the ACC. ACC has however also been hypothesised to have a potential to improve safety and to reduce the environmental impact of road traffic. These aspects of traffic are to a larger extent than quality-of-service dependent on the movements of individual vehicles in the traffic stream. Large acceleration and deceleration rates can for example lead to both safety critical situations and increased fuel consumption and pollutant emissions.

Individual vehicle movements can be described by position, speed and acceleration time series. Such time series are commonly referred to as vehicle trajectories. The aim of this paper is to use vehicle trajectories from traffic simulation to quantify potential impacts of ACC on vehicle acceleration and deceleration rates. The analysis is based on traffic simulations of mixed traffic including ACC-equipped and standard vehicles. Car-following models that include ACC system functionality and driver behaviour in ACC-equipped as well as standard vehicles are used in the simulations. We also investigate how the results are dependent on driver 
behaviour in terms of desired speeds, desired following gaps and reaction times. The purpose of this work is not to evaluate a specific ACC system but to provide knowledge of potential vehicle trajectory impacts of ACC functionality and driver behavioural adaptations related to ACC.

The remainder of this paper is organised as follows. Section 2 presents properties of ACC systems including system functionalities and observed changes in driver behaviour. Requirements on car-following models used for simulation of traffic including ACC-equipped vehicles are also discussed. Section 3 presents the used car-following models for ACC-equipped and standard vehicles and presents an indicator of vehicle trajectory smoothness. Vehicle trajectory impacts of ACC are then studied in Section 4 before conclusions and suggestions for further research are given in Section 5.

\section{Adaptive cruise control and car-following}

Adaptive Cruise Control (ACC) was one of the first ADAS to be introduced on the market. ACC extends the functionality of traditional fixed speed cruise control with a distance controller. If there are no vehicles within following distance in-front of the ACC-equipped vehicle then ACC works as a fixed speed cruise control and adjusts the vehicle's speed towards a desired speed set by the driver. In situations with a slower vehicle within following distance of the ACC-equipped vehicle, the distance controller of the ACC system regulates the distance to the leader vehicle. A fixed time strategy is commonly adopted, i. e. the driver sets a desired time gap to the leader vehicle and the ACC updates the vehicle's acceleration/deceleration to maintain this desired time gap. When driving with the ACC system active, the driver remains responsible for the drive and may resume control at any time. The operation of many early ACC systems is limited to speeds corresponding to free flow traffic conditions. The acceleration/deceleration used by the ACC is also commonly restricted to a sub-range of the vehicle's capability. ACC systems applicable to all speeds, including "stop-and-go" traffic, are however expected in the near future. The ACC system functionality can, based on the given description, be described as a distance and speed controller.

Improved driver comfort is the main reason for the development and introduction of ACC. It has however been hypothesised that ACC could have an impact on road safety and quality-of-service (Golias et al., 2002). The reasoning behind these hypotheses is that as the ACC distance 
controller has a potential to be a more efficient regulator than a human driver, the number of rear-end collisions can be reduced and vehicles can travel closer together at higher speeds and thereby increase the capacity of the road. There may also be environmental gains to be made from increased ACC usage. More efficient following distance control facilitates the use of lower acceleration and deceleration rates which in turn reduces fuel consumption and vehicle emissions.

ACC is one of the most studied ADAS in terms of the system's impacts on driver behaviour. Many studies have focused on concerns raised about driver behavioural adaptation in relation to driving with ACC. There are apprehensions about if the reduction in driver workload may reduce attention to the driving task. The driver's ability to deal with the limitations of the ACC system and to resume control in critical situations has also been frequently discussed. Driving simulator studies have confirmed that drivers react later to critical situations when driving with ACC (Saad et al., 2004). The observed delays were attributed to either reduced attention or over-reliance on the ACC. A reduction in driver workload has also been established (Dragutinovic et al., 2005). Commonly observed changes in the continuous driving behaviour in ACC-equipped vehicles are changes in speeds and car-following time headways.

Numerous traffic simulation studies of ACC can also be found in the literature (Minderhoud and Bovy, 1999; Hoogendoorn and Minderhoud, 2002; Davis, 2004, 2007; van Arem et al., 2006; Kesting et al., 2007a, 2007b). Traffic simulation has traditionally been applied for quality-of-service analysis of proposed changes in traffic management or road network design. Most traffic simulation studies of ACC are for this reason focused on macroscopic quality-of-service effects, such as changes in road capacity and impacts on average speeds. Driver behavioural adaptations are also usually not considered. This simplification may be justified for analyses based on macroscopic quality-of-service indicators. Changes in driver behaviour can however be crucial for analyses that rely on representative vehicle trajectories. There is consequently a need for analysis of the sensitivity of traffic simulation based vehicle trajectories to the assumptions made regarding driver behaviour.

An ACC system influences the longitudinal movements of the equipped vehicle. Longitudinal vehicle movements are in a traffic simulation model governed by a car-following model. It is therefore necessary to modify the car-following model to allow simulation of ACC vehicles. 
Many commonly applied car-following models are in essence controllers that determine acceleration/deceleration rates given distance and speed difference to the immediate leader (Janson Olstam and Tapani, 2003). Hence, car-following models and models of ACC systems have the same input data. ACC system functionality can therefore straightforwardly be taken into account by changing parameters or functional form of the car-following model. The car-following model should also include parameters controlling speeds and car-following headways to allow modelling of observed driver behavioural changes. Delayed reactions in situations when the driver need to resume control of the longitudinal driving task should also be modelled if such situations are to be studied. The importance of this aspect is however likely to decrease as "stop-and-go" ACC systems are introduced.

More important for the analysis of future impacts of ACC is probably the modelling of non-equipped vehicles in simulations of mixed traffic. The most obvious difference between ACC and human driving is the longer reaction time of human drivers. Human drivers are also likely to estimate the position and speed of the leader vehicle with less accuracy than an ACC system's sensors. Limited perception capabilities are however compensated for by the drivers through anticipation of future traffic situations. This anticipation can, to be more precise, be described as consideration of multiple vehicles ahead and accounting for future speeds and positions.

The above described aspects need to be considered in detailed carfollowing modelling of human drivers. However, Treiber et al. (2006) showed that macroscopic traffic dynamics resulting from simulations with a detailed car-following model including the human limitations discussed above were equal to the conditions resulting from simulations with a simpler, and more ACC like, car-following model. This result indicates that, when considering macroscopic traffic flow dynamics, anticipation and human limitations cancel out. It is consequently appropriate to use a car-following model with less modelling detail for macroscopic quality-of-service analysis. There will nevertheless be differences in the underlying vehicle trajectories for simulations using different levels of car-following modelling detail. These differences, which may prove to be important for detailed analyses based on entire vehicle trajectories, are investigated in the remainder of this paper. 


\section{Traffic simulation of ACC-equipped and standard vehicles}

This paper presents results of traffic simulations including ACC-equipped and standard vehicles. The car-following models applied for these simulations are described in this section. The objective of the simulations is to study impacts of ACC on vehicle acceleration and deceleration rates. The utilised indicator of vehicle acceleration trajectory smoothness is also presented in this section.

All simulations were performed using the Rural Traffic Simulator (RuTSim) framework presented by Tapani (2005). RuTSim is a stochastic simulation model and simulation runs based on different random number generator seed will consequently give different results. For this reason, all results are given as confidence intervals based on repeated simulation runs using five different random number generator seeds. This number of repetitions was found to give reasonably narrow confidence intervals for most of the simulated situations. The use of an equal number of random number generator seeds for all simulations also allow differences in the stochasticity of the resulting traffic conditions to be studied through the confidence interval widths.

The simulated road is a one-directional open traffic system including a $500 \mathrm{~m}$ long one-lane section followed by a $1000 \mathrm{~m}$ long two-lane section completed by a lane drop and a $500 \mathrm{~m}$ long one-lane section. This road was modelled to allow studies of simulated vehicle trajectories under the influence of a lane drop bottle neck.

\subsection{Car-following modelling of ACC and human drivers}

The basic car-following model used to model both ACC and standard vehicles is the Intelligent Driver Model (IDM) introduced by Treiber et al. (2000). The IDM determines a vehicle's acceleration rate at time $t$ according to

$$
a(t)=a_{0}\left[1-\left(\frac{v(t)}{v_{0}}\right)^{4}-\left(\frac{s^{*}(v(t), \Delta v(t))}{s(t)}\right)^{2}\right],
$$

where $v(t)$ and $v_{0}$ are the current and desired speed, respectively, of the considered vehicle, $\Delta v(t), s(t)$ and $s^{*}(v(t), \Delta v(t))$ are the approach speed, the current gap and the desired gap to the vehicle in-front, respectively, and $a_{0}$ is a parameter that determine maximum acceleration. 
The desired gap is given by

$$
s^{*}(v(t), \Delta v(t))=s_{0}+v(t) T_{d}+\frac{v(t) \Delta v(t)}{2 \sqrt{a_{0} b}},
$$

where $s_{0}$ is the minimum distance between vehicles, $T_{d}$ is the desired steady state time gap and $b$ is a parameter that correspond to the desired maximum deceleration rate. In the simulations, parameter values presented by Kesting et al. (2007a) have been used for all vehicles, i. e. $T_{d}=1.5 \mathrm{~s}, a_{0}=1.4 \mathrm{~m} / \mathrm{s}^{2}, b=2 \mathrm{~m} / \mathrm{s}^{2}$ and $s_{0}=2 \mathrm{~m}$.

For standard vehicles, limited perception and reaction capabilities and anticipation to compensate for these limitations have been taken into account by extending the IDM model with the Human Driver Model (HDM) presented by Treiber et al. (2006). HDM is a meta-model that allows modelling of delayed reactions, perception inaccuracies and anticipation of the future traffic situation. The ACC system response time was also taken into account.

The reaction delay, $T^{\prime}$, is in the HDM modelled by evaluating the left hand side of Equation 1 at time $t$ and the right hand side at time $t-T^{\prime}$, i. e. one reaction delay time earlier. The reaction delay have been set to $1.2 \mathrm{~s}$ for standard cars and to $0.1 \mathrm{~s}$ for ACC cars.

The HDM take into account inaccuracies in a driver's estimation of the approach speed and the distance to the leader. Imperfect estimation of the approach speed to the lead vehicle are modelled by assuming that the uncertainty of the estimation of $\Delta v$ is proportional to the distance $s$, i. e. drivers estimate the time-to-collision, $s / \Delta v$, with constant uncertainty. This assumption is supported by empirical observations. Persistence of the estimation errors are accounted for by modelling the error as a Wiener process. Then the estimated approach speed can be expressed as

$$
(\Delta v)^{e s t}(t)=\Delta v(t)+s(t) r_{c} w_{\Delta v}(t)
$$

where $r_{c}$ is the inverse of the average estimation error of time-to-collision and $w_{\Delta v}(t)$ is a Wiener process with unit variance and correlation time $\tau$. The uncertainty in the estimation of the distance to the leader is defined in a relative way by assuming a constant variation coefficient, $V_{s}$. As for the estimation error for the approach speed, the error is modelled as a Wiener process with correlation time $\tau$. The estimated distance to the leader can be expressed as

$$
s^{e s t}(t)=s(t) e^{V_{s} w_{s}(t)},
$$


where $w_{s}(t)$ is a Wiener process with unit variance and correlation time $\tau$. The Wiener processes $w_{\Delta v}(t)$ and $w_{s}(t)$ are independent. Simulation of the Wiener processes was conducted using the numerical scheme proposed by Treiber et al. (2006). The HDM driver perception error parameters were also set to the values presented by Treiber et al. (2006), i. e. $r_{c}=0.01 \mathrm{~s}^{-1}, V_{s}=5 \%$ and $\tau=20 \mathrm{~s}$.

The HDM also take into account driver anticipation to compensate for the reaction delay. A "constant acceleration" heuristic is adopted for the anticipation of the future speed, $v^{\prime}(t)$. The future speed is obtained as

$$
v^{\prime}(t)=v\left(t-T^{\prime}\right)+T^{\prime} a\left(t-T^{\prime}\right) .
$$

Other vehicles acceleration is known to be difficult to estimate for human drivers. A "constant speed" heuristic is therefore adopted for the anticipation of the future distance, $s^{\prime}(t)$, and approach speed, $\Delta v^{\prime}(t)$, to the leader. We obtain

$$
s^{\prime}(t)=s^{e s t}\left(t-T^{\prime}\right)-T^{\prime} \Delta v^{e s t}\left(t-T^{\prime}\right)
$$

and

$$
\Delta v^{\prime}(t)=\Delta v^{e s t}\left(t-T^{\prime}\right)
$$

The anticipated future speed, distance and approach speed given by Equations 2-4 are used as input to the IDM model in Equation 1.

The final aspect of human driving considered in HDM is spatial anticipation by considering multiple vehicles ahead. This is accomplished by separating the right hand side of Equation 1 into free driving acceleration

$$
a^{\text {free }}=a_{0}\left[1-\left(\frac{v(t)}{v_{0}(t)}\right)^{4}\right]
$$

and interaction acceleration

$$
a^{i n t}=-a_{0}\left(\frac{s^{*}(v(t), \Delta v(t))}{s(t)}\right)^{2} .
$$

Interaction with $n$ leaders are modelled by summation of the interaction accelerations between the considered vehicle, $i$, and the nearest $n$ vehicles. Then, the acceleration rate of vehicle $i$ can be expressed as

$$
a_{i}(t)=a_{i}^{f r e e}+\sum_{j=i-n}^{i-1} a_{i j}^{i n t},
$$


where $a_{i}^{\text {free }}$ is given by Equation 5 and $a_{i j}^{i n t}$ is given by Equation 6 where $i$ is the considered vehicle and $j$ is the leader that is taken into account. Drivers in standard cars have been assumed to consider three vehicles infront in car-following situations, i. e. we set $n=3$. Car-following models including three leaders have been found to fit empirical data better than models including fewer vehicles in-front (Hoogendoorn et al., 2006).

\subsection{An indicator of vehicle trajectory smoothness}

Acceleration noise is suggested by Leutzbach (1988) as a good indicator of the smoothness of the traffic flow. For a single vehicle $i$ it is defined as the standard deviation of acceleration according to

$$
\mathrm{ACN}_{i}=\sqrt{\frac{1}{T} \int_{0}^{T}[a(t)-\bar{a}] \mathrm{d} t},
$$

where $T$ is the travel time over which $\mathrm{ACN}_{i}$ is calculated, $a(t)$ is the vehicle's acceleration rate at time $t$ and $\bar{a}$ is the average acceleration computed as

$$
\bar{a}=\frac{1}{T} \int_{0}^{T} a(t) \mathrm{d} t .
$$

In this work, $\mathrm{ACN}_{i}$ is applied as an indicator of the smoothness of the simulated vehicle trajectories. Large values of $\mathrm{ACN}_{i}$ indicates large changes in acceleration and deceleration which in turn may decrease road safety and increase the environmental impacts of traffic. Conversely, low values of $\mathrm{ACN}_{i}$ indicates a smoothly travelling vehicle. $\mathrm{ACN}_{i}$ is an individual vehicle indicator. In the simulation we use ACN defined as the average of $\mathrm{ACN}_{i}$ over all vehicles as an indicator of the collective situation.

Congested traffic implies larger and more frequent acceleration and deceleration changes. $\mathrm{ACN}_{i}$ will consequently increase with increasing traffic flow. Figure 1 shows the resulting ACN from simulations with varying input traffic flows. All simulated flows contained only standard vehicles. As can be seen in the figure, $\mathrm{ACN}$ is increasing with increasing traffic flow. This is in accordance with the expectation. Increasing stochasticity caused by more vehicle interactions in higher flows gives rise to larger differences between different simulation runs as indicated by the wide confidence interval for the highest flow. Simulations of traffic flows close to the capacity of the lane drop bottle neck, i. e. 1400 to 1500 


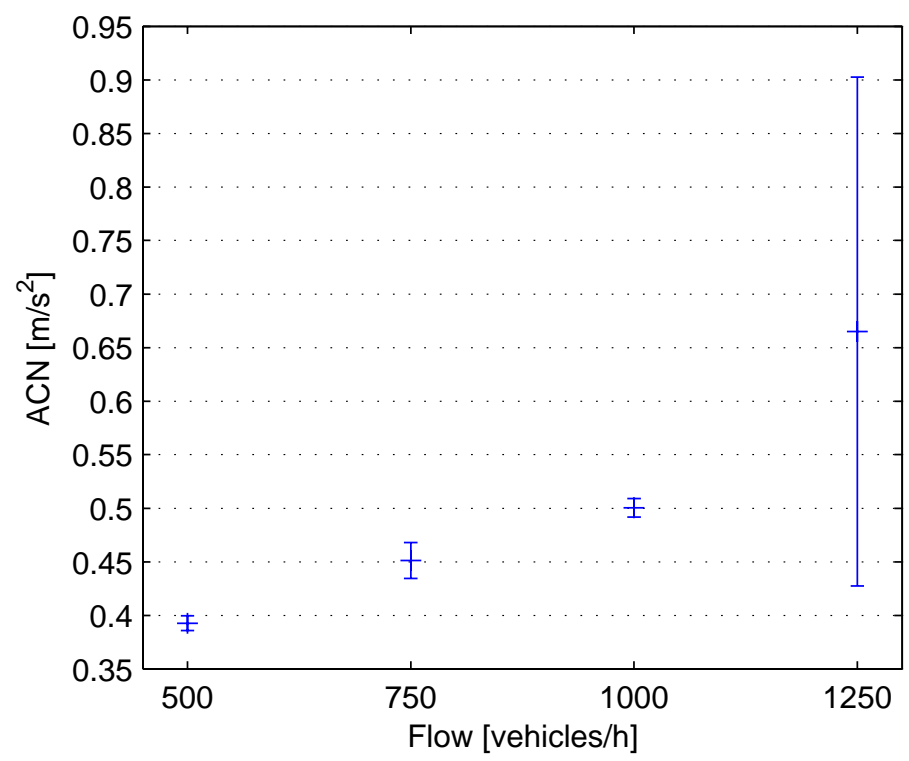

Figure 1: Average ACN for varying traffic flows, $95 \%$ confidence intervals

vehicles per hour, confirm these observations. ACN grows very fast with increased flow and the corresponding confidence intervals become very wide.

A total flow of 1000 vehicles per hour was chosen for the remaining simulations based on these observations. This flow results in significant vehicle interactions without increasing the stochasticity of the traffic flow. Resulting confidence intervals will consequently be narrow enough to allow conclusions to be drawn with a reasonable number of repetitions of the simulations.

\section{Impacts of ACC, human limitations and driver behaviour}

Impacts of driver behavioural adaptation to ACC and the effects of detailed car-following modelling of human limitations are in this section studied through traffic simulations of a road section with a mix of standard and ACC-equipped cars.

The modelled ACC system is assumed to be operative at all speeds 
and drivers in ACC-equipped vehicles are assumed to use the ACC at all times. System limitations and driver acceptance of the ACC are consequently not considered. In addition, drivers in ACC-equipped and standard vehicles are as a starting point of the analysis assumed to have equal desired following time gaps and desired speeds. Consequences of this assumption are investigated as part of the subsequent analysis.

\subsection{Vehicle trajectory impacts of ACC}

The difference in the applied car-following modelling of standard and ACC cars are the following. Human reaction delays, perception inaccuracies and anticipation are taken into account in the model used for standard cars and the model for ACC-equipped cars assume a short system response time. The impacts of these aspects on ACN have been studied through simulations of mixed traffic including varying percentages of ACC vehicles. Simulations of traffic with varying ACC penetration rate also allowed analysis of changes in traffic conditions as the percentage of ACC-equipped vehicles grows. One expects that the faster and more accurate response of the ACC system will result in smoother trajectories for ACC cars. This was also confirmed by the simulation results as shown in Figure 2. A tendency towards decreased ACN and smoother trajectories for both standard and ACC cars with increased percentages of ACC cars could also be observed. This effect is likely due to that a vehicle behind an ACC vehicle will have more time to respond to changes in speed of the downstream platoon. A certain percentage of ACC vehicles in the traffic stream could therefore prevent and reduce the severity of shock waves. This conclusion has also been drawn from the traffic simulation based capacity and quality-of-service studies of ACC referred to in Section 2.

\subsection{Sensitivity of the results to changes in driver behaviour}

The aim of this paper is to use traffic simulation based vehicle trajectories to quantify impacts of ACC on vehicle acceleration and deceleration rates and to investigate the influence of the assumed driver behaviour on the results. Observed behavioural adaptations of drivers in ACCequipped vehicles include changes in following time gaps and speeds. Impacts of these changes have been studied by simulations of traffic including $20 \%$ ACC cars. Positive effects of ACC can to a large extent be 


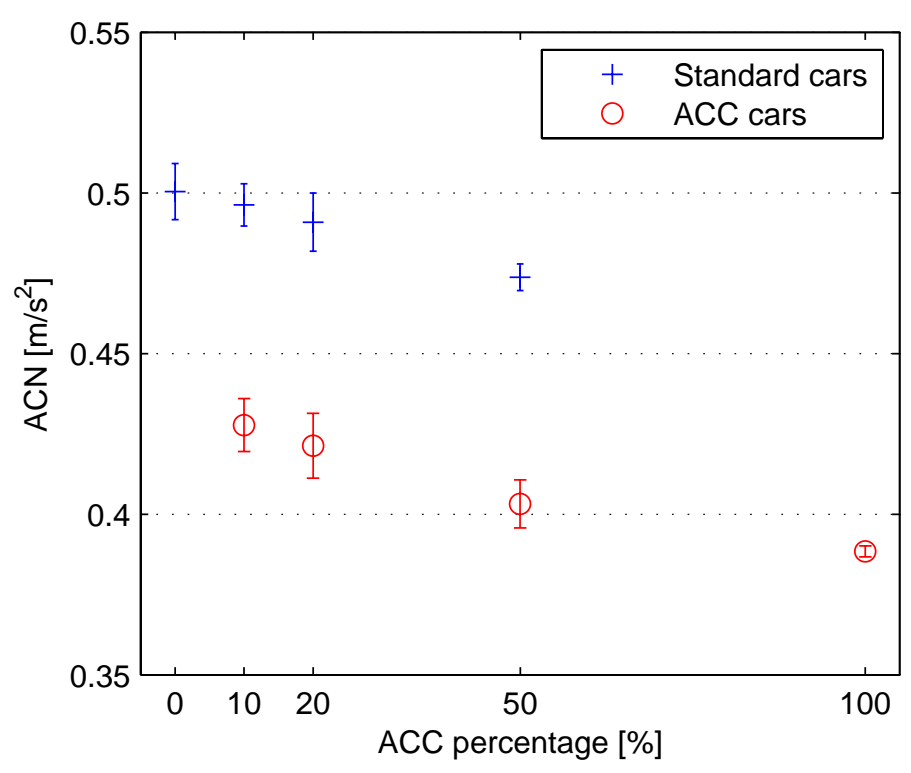

Figure 2: Average ACN for traffic with varying percentages of vehicles equipped with ACC, $95 \%$ confidence intervals

contributed to the faster reaction of the ACC system compared to a human driver. The impact of the delayed reactions of drivers in standard cars has also been investigated.

The first experiment is concerned with the impact of changed following time gaps for ACC-equipped vehicles. Reduced following time gaps can be expected to lead to increased acceleration and deceleration rates. Following time headways in the IDM model are controlled by the desired steady state time gap, $T_{d}$. ACN from simulations with varying $T_{d}$ for ACC cars are shown in Figure 3. The desired following time gaps of standard cars have been kept constant at $1.5 \mathrm{~s}$ in the simulations. As expected, reduced desired following time gap starting from $1.5 \mathrm{~s}$ for ACC cars lead to increased ACN. This effect is small and non-equipped standard cars seem to be uninfluenced. However, for the shortest time gap setting the trajectories of the standard cars become unstable. The corresponding large ACN and wide confidence interval does not fit the scale of the other results and are therefore not included in the figure. This instability is likely caused by the reduction of the time and space available for reactions when travelling behind an ACC car that travels 


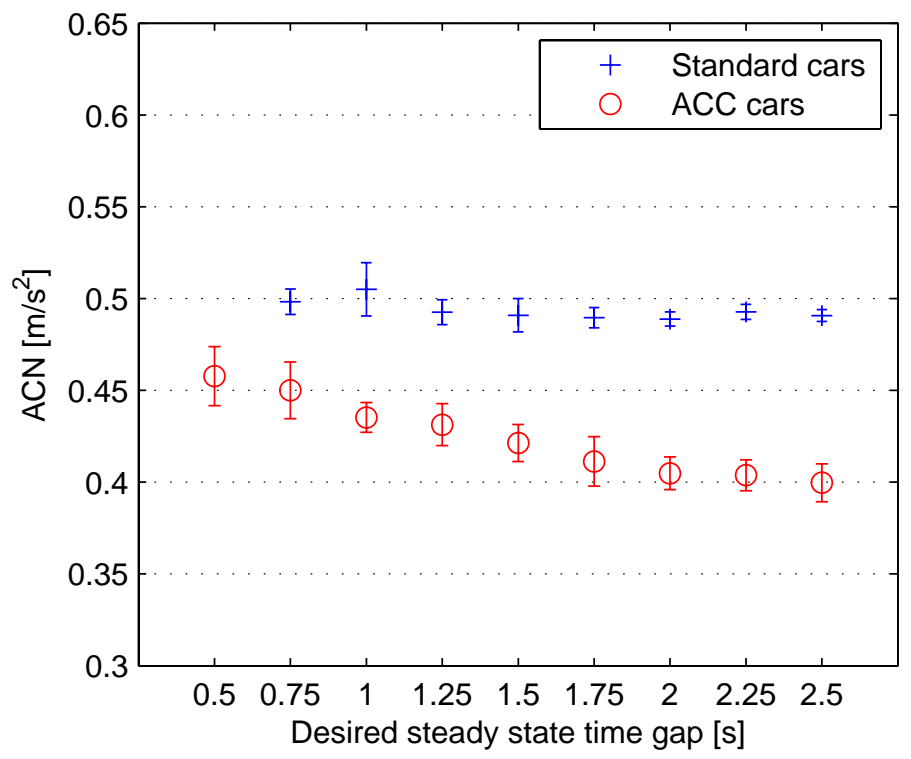

Figure 3: Average ACN of standard and ACC equipped cars for varying desired following time gap of the ACC equipped cars, $95 \%$ confidence intervals 


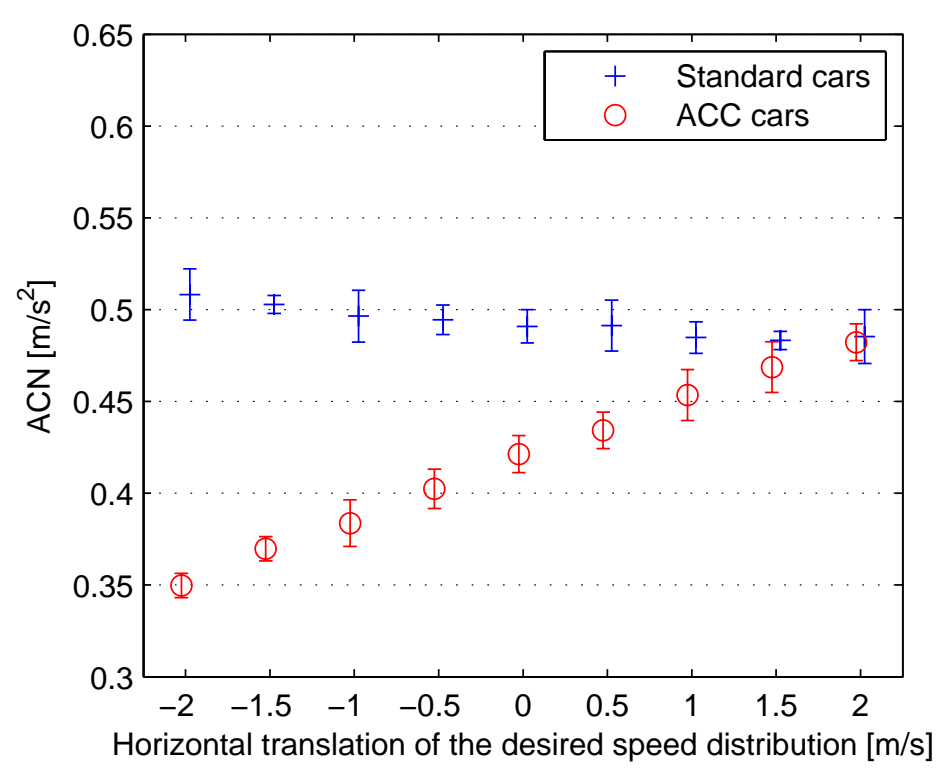

Figure 4: Average ACN of standard and ACC equipped cars for varying horizontal translation of the desired speed distribution of the ACC equipped cars, $95 \%$ confidence intervals

close to its leader. The impact of increased desired following time gaps for ACC cars starting from $1.5 \mathrm{~s}$ is smaller than the impact for reduced following time gaps. This is likely due to that as the following time gaps increase the influence on $\mathrm{ACN}$ of vehicle-to-vehicle interactions during car-following decrease.

The second experiment is concerned with the impact of changed desired speeds of drivers in ACC-equipped vehicles. Both ACC and standard cars have in the previously described simulations been assigned desired speeds from RuTSim's default desired speed distribution for cars. Simulations with varying desired speeds of the ACC cars have been performed to study the impact of changes in speed when driving with ACC. Modification of the desired speeds of ACC cars has been carried out by horizontal translation of the corresponding desired speed distribution. The distribution mean has been shifted and the variance is kept constant. The results of these simulations are shown in Figure 4. Vehicles with lower desired speed can be assumed to interact less with vehicles infront. Less interaction implies less accelerations and decelerations and 


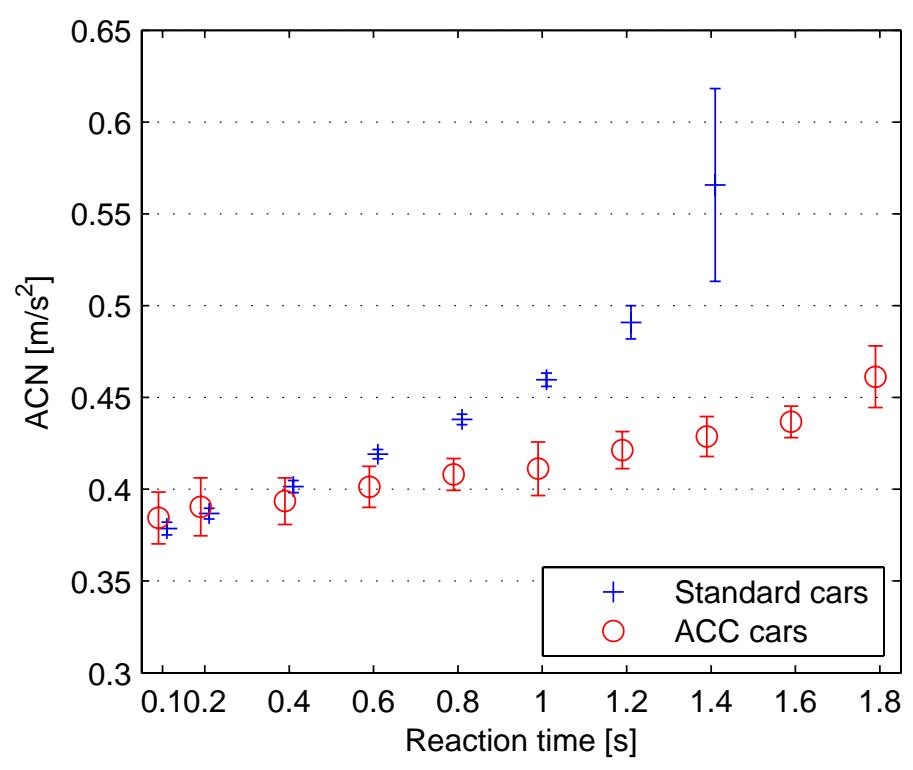

Figure 5: Average ACN of standard and ACC equipped cars for varying reaction time of the standard cars, $95 \%$ confidence intervals

thereby lower ACN. This is confirmed by the simulation results in Figure 4. Conversely, vehicles with higher desired speeds will be constrained by slower vehicles more often and therefore accelerate and decelerate more. This can also be observed in the results for positive shifts of the desired speed distribution for ACC cars. Shift of the desired speed distribution of ACC cars with $1.5 \mathrm{~m} / \mathrm{s}$ results in inseparable ACN for standard and ACC cars. It is also possible to conclude that an ACC system will result in less positive vehicle trajectory impacts the higher the desired speeds of the equipped vehicles.

The third experiment is concerned with the impact of the delayed reactions of drivers in standard cars. Positive traffic system effects of ACC can to large extents be related to the faster reaction of the ACC system compared to a human driver. It is consequently interesting to study the importance of the delayed reactions of drivers in standard cars. It can be expected that reduced driver reaction times will lead to smoother vehicle trajectories. Figure 5 shows the results of simulations with varying reaction times of standard cars. The results confirm that reduced driver reaction times lead to decreased ACN. A reaction time 
for standard cars of below $0.4 \mathrm{~s}$ was found to result in inseparable ACN for standard and ACC cars. The assumed ACC system response time is $0.1 \mathrm{~s}$. It is therefore possible to conclude that the modelled human driver behaviour in standard cars correspond to a reduction in reaction time of approximately $0.3 \mathrm{~s}$. The modelled human driver behaviour aspects consist of anticipation, which can be expected to increase trajectory smoothness, and estimation inaccuracies, which will have a negative impact on trajectory smoothness. Anticipation of the future traffic situation can therefore compensate for more than $0.3 \mathrm{~s}$ longer reaction time. Detailed analyses of impacts of driver anticipation and inaccuracies on vehicle trajectory smoothness are interesting topics for further research.

Increased driver reaction times were, in accordance with the expectation, found to increase ACN. The trajectories of standard cars become unstable for reaction times over $1.4 \mathrm{~s}$. This result is natural since the assumed desired following time gap is set to $1.5 \mathrm{~s}$. A consequence of this instability are very large ACN that are outside the scale of the other results. These large values are therefore not shown in Figure 5. An impact on the trajectories of ACC cars can also be established. The effect seems to increase as the reaction time of standard cars increase. This can be explained as a consequence of interactions between standard and ACC cars in the traffic stream. As the accelerations and decelerations of standard cars increase, ACC cars are also forced to use increased acceleration and deceleration rates in order to prevent collisions. Noticeable is however the bounded ACN of ACC cars. This indicates that the trajectories of ACC cars remain stable when the trajectories of standard cars become unstable. ACC will therefore have a stabilising effect on the traffic stream. Further analysis of this effect could be conducted through simulations of varying driver reaction times in traffic with different percentages of ACC cars.

\section{Conclusions}

The aim of this paper is to use trajectories from traffic simulation to study impacts of ACC on vehicle acceleration and deceleration rates. The analysis is based on simulations of traffic including ACC-equipped and standard cars. As a basic scenario, the simulated ACC system was assumed not to have any influence on drivers' desired speeds or following time headways. Such an ACC-system have previously been found to have no impact on macroscopic road capacity (Minderhoud and Bovy, 
1999). In addition, Treiber et al. (2006) showed that simulations with an ACC-like car-following model resulted in equal macroscopic traffic dynamics as simulations with a more complex human driver model. However, the results of this work show that ACC-equipped vehicles use lower acceleration and deceleration rates than standard non-equipped vehicles. This indicates that ACC may have positive consequences for safety and the environmental impact of road traffic even though the macroscopic traffic conditions remain unchanged. Moreover, non-equipped standard vehicles were found to be positively influenced by increasing percentages of ACC vehicles in the traffic stream.

We have also shown that the impacts of ACC are to large extents dependent on the assumptions made regarding driver behaviour. This finding was established through simulations with varying desired following time gaps and desired speeds of ACC-equipped vehicles. Changes in desired speeds and following time gaps are commonly reported from driver behavioural studies of ACC (Dragutinovic et al., 2005; Saad et al., 2004). Simulations with varying reaction times of standard vehicles were also performed. These simulations confirmed that the positive impacts of ACC can largely be contributed to the faster reaction of the ACC system compared to a human driver. The results reported on in this paper show that it is crucial to include appropriate assumptions on driver behaviour in the analysis. Notably, this aspect have usually not been taken into account in previous traffic simulation based studies of ACC.

Further research related to the work presented in this paper include quantification of environmental and road safety impacts of ACC. The presented findings on properties of simulated vehicle trajectories will provide a basis for this work. There is however a general need to relate traffic simulation based performance indicators other than macroscopic quality-of-service measures to empirically observed effects. It is also of interest to investigate the impact of the lane-changing modelling on the smoothness of traffic simulation based vehicle trajectories.

\section{Acknowledgements}

This work was sponsored by the Swedish Road Administration through the Swedish network of excellence Transport Telematics Sweden. The author would like to thank Jan Lundgren, Linköping University for priceless support during the entire research process. 


\section{References}

Berghout, L., E. Versteegt, B. van Arem, R. Naomi, and G. Bootsma (2003). Advanced driver assistance systems; results of the state of the art of ADASE-II. ADASE-II deliverable D2A, ADASE 2 Consortium, Brussels.

Davis, L. C. (2004). Effect of adaptive cruise control systems on traffic flow. Physical Review E 69, 066110.

Davis, L. C. (2007). Effect of adaptive cruise control systems on mixed traffic flow near an on-ramp. Physica A 379, pp. 274-290.

Dragutinovic, N., K. A. Brookhuis, M. P. Hagenzieker, and V. A. W. J. Marchau (2005). Behavioural effects of advanced cruise control use a meta-analytic approach. European Journal of Transport and Infrastructure Research 5(4), pp. 267-280.

Golias, J., G. Yannis, and C. Antoniou (2002). Classifcation of driverassistance systems according to their impact on road safety and traffic efficiency. Transport Reviews 22(2), pp. 179-196.

Hoogendoorn, S. P. and M. M. Minderhoud (2002). Motorway flow quality impacts of advanced driver assistance systems. Transportation Research Record 1800, pp. 69-77.

Hoogendoorn, S. P., S. Ossen, and M. Schreuder (2006). Empirics of multianticipative car-following behavior. Transportation Research Record 1965, pp. 112-120.

Janson Olstam, J. and A. Tapani (2003). Comparison of car-following models. VTI meddelande 960A, Swedish National Road and Transport Research Institute, Linköping.

Kesting, A., M. Treiber, M. Schönhof and D. Helbing (2007a). Extending adaptive cruise control to adaptive driving strategies. Transportation Research Record 2000, pp. 16-24.

Kesting, A., M. Treiber, M. Schönhof, F. Kranke and D. Helbing (2007b). Jam-avoiding adaptive cruise control (ACC) and its impact on traffic dynamics. In A. Schadschneider, T. Pöschel, R. Kühne, M. Schreckenberg, and D. E. Wolf (Eds.), Traffic and Granular Flow '05. Berlin Heidelberg: Springer-Verlag. 
Leutzbach, W. (1988). Introduction to the Theory of Traffic Flow. Berlin Heidelberg: Springer-Verlag.

Liu, R. and J. Tate (2004). Network effects of intelligent speed adaptation systems. Transportation 31, pp. 297-325.

Minderhoud, M. M. and P. H. L. Bovy (1999). Impact of intelligent cruise control on motorway capacity. Transportation Research Record 1679, pp. 1-9.

Saad, F., M. Hjälmdahl, J. Cañas, M. Alonso, P. Garayo, L. Macchi, F. Nathan, L. Ojeda, V. Papakostopoulos, M. Panou, and E. Bekiaris (2004). Literature review of behavioural effects. AIDE deliverable D1.2.1, Information Society Technologies, Brussels.

Tapani, A. (2005). Versatile model for simulation of rural road traffic. Transportation Research Record 1934, pp. 169-178.

Tapani, A. (2008). Analysis of rumble strips and driver fatigue using traffic simulation. Advances in Transportation Studies 14, pp. 69-80.

Treiber, M., A. Hennecke, and D. Helbing (2000). Congested traffic states in empirical observations and microscopic simulations. Physical Review E 62(2), pp. 1805-1824.

Treiber, M., A. Kesting, and D. Helbing (2006). Delays, inaccuracies and anticipation in microscopic traffic models. Physica A: Statistical Mechanics and its Applications 360, pp. 71-88.

van Arem, B., C. J. G. van Driel, and R. Visser (2006). The impact of cooperative adaptive cruise control on traffic-flow characteristics. IEEE Transactions on Intelligent Transportation Systems 7(4), pp. 429-436. 
